\title{
CONSIDERATIONS REGARDING COMETARY AND INTERSTELLAR MOLECULES*
}

\section{P. SwINGS ${ }^{I}$}

\section{ABSTRACT}

The presence of $\mathrm{CH}^{+}$molecules in comets is demonstrated by the attribution of three cometary lines, $\lambda \lambda$ 423I 4238.5 , and 4254.4, to the first transitions of the three branches of the $(0,0)$ band. There is also good evidence for the presence of the $(\mathrm{I}, \mathrm{O})$ band of $\mathrm{CH}^{+}$. The $\mathrm{CH}^{+}$molecules are observed in the head and in part of the tail.

The spectral region $\lambda \lambda 3560-3600$ of cometary spectra is discussed. It seems very probable that some of the features are not due to the $\Delta v=+$ I sequence of $C N$ and to the $(8,0)$ band of $C O^{+}$and that the $(\mathrm{o}, \mathrm{o})$ band of $\mathrm{OH}^{+}$plays some role. But spectrograms of higher resolving-power are required to settle this matter.

Additional evidence is given for the presence of the isotopic bands of $C_{2}$ in cometary spectra; the $C^{12} C^{13}$ molecule is observed in the three transitions $(\mathrm{I}, \mathrm{O}),(2, \mathrm{I})$, and $(3,2)$.

The problems of molecular formation, dissociation, and ionization are quite different in interstellar space and in comets. In interstellar space the main source of molecular formation is by two-body encounters with simultaneous emission of radiation. In comets the diatomic molecules result from the liberation of occluded gases and from the photodissociation of more complex molecules.

The dissociation equilibrium of $\mathrm{CH}^{+}$in interstellar space is discussed. Account has to be taken of the fact that practically all the $C H^{+}$molecules are on the lowest rotational level. Thus the photodissociation may arise only from this lowest level, whereas the formation by capture may lead to any excited level. The corresponding correction factor has a value of the order of IO $^{3}$. The computed abundance of $\mathrm{CH}^{+}$ is in agreement with the observations.

The ionization equilibrium of $\mathrm{CH}^{+}$and $\mathrm{CH}$ is considered. It is suggested that the electron density in the regions of molecular absorption is about $10^{-x}$ electron per cubic centimeter.

The dissociation and ionization equilibrium of $C N$ cannot yet be discussed because of the absence of essential physical data, especially the ionization potential of $C N$. The abundance of $C_{2}$ molecules must be much lower than that of $C N$ or $C H$; moreover each $\left(v^{\prime}, 0\right)$ band of the interstellar $C_{2}$ molecules would possess a large number of absorption lines, instead of three or less as in the case of $C H, C H^{+}$, and $C N$. From these considerations it is concluded that interstellar lines of $C_{2}$ must be very much weaker than those of $\mathrm{CH}, \mathrm{CH}^{+}$, and $\mathrm{CN}$.

Molecules of $\mathrm{CH}$ and $\mathrm{CN}$ are observed both in the comets and in interstellar gas, whereas $C_{2}$, which is abundant in cometary heads, has not been found in interstellar absorption. ${ }^{2}$ In this paper a comparative discussion is made of the mechanisms of molecular formation, dissociation, and ionization in comets and in interstellar space. Such a discussion requires additional identification work which constitutes the subject of the first section of the paper.

\section{IDENTIFICATION PROBLEMS}

In addition to the ten atoms and ions ${ }^{3}-\mathrm{H}, \mathrm{O}^{+}, \mathrm{O}^{++}, \mathrm{N}^{+}, \mathrm{Na}, \mathrm{Ca}, \mathrm{Ca}^{+}, \mathrm{Ti}^{+}, \mathrm{K}, \mathrm{Fe}-$ three molecules have been definitely found in interstellar space; these are $C N, C H$, and $\mathrm{CH}^{+}$. Still unidentified are two sharp interstellar absorption lines at $\lambda 3579.04$ and

* Contributions from the Lick Observatory, University of California, Ser. II, No. 3 .

I Alexander F. Morrison Research Associate in the Lick Observatory.

${ }^{2}$ For a general account of the problem of the interstellar molecules see A. McKellar, Pub. Dom. Ap. Obs., in press. I am greatly indebted to Dr. McKellar for the privilege of reading his important paper in advance of publication. See also P. Ledoux, Pop. Astr., 49, 513, r94I (summary of the symposium on interstellar lines at the Yerkes Observatory, on June 30, I94I); P. Swings, J.R.A.S., Canada, 35, 8I, I94I; Ann. d'ap., I, 39, I938.

${ }^{3} \mathrm{H}, \mathrm{O}^{+}, \mathrm{O}^{++}$, and $\mathrm{N}^{+}$have been revealed by their emission lines, the other elements (atoms or molecules) by their absorption lines. 
$\lambda$ 3934.294 and a set of seven broad features: one about $40 \mathrm{~A}$ wide at $\lambda 4430$, the six others $^{5}$ about $3 \mathrm{~A}$ wide at $\lambda \lambda_{5780.55}$ (int. 3), 5797. I 3 (int. I), 6202.99 (int. $\mathrm{I}^{-}$), 6269.99 (int. I), 6283.9 (int. 6), and 6613.9 (int. 2).

In cometary atmospheres seven molecules have been definitely identified: $O H, N H$, $\mathrm{CN}, \mathrm{CH}, \mathrm{C}_{2}, \mathrm{CO}^{+}$, and $\mathrm{N}_{2}^{+}$, but there are still a number of unidentified features.

Since the physical conditions in comets are such that the ionization of molecules is possible, a discussion of other ionized diatomic molecules appears promising. Unfortunately, such a discussion has to be limited at the present time to $\mathrm{CH}^{+}, \mathrm{OH}^{+}$, and $\mathrm{O}_{2}^{+}$, since these are the only three ionized molecules consisting of cosmically abundant atoms that have been investigated in the laboratory. Additional experimental work is urgently needed, since it appears quite probable that the strong unidentified group of lines near

TABLE 1

GROUP OF UNIDENTIFIED COMETARY LINES NEAR $\lambda 4240$

\begin{tabular}{|c|c|c|c|c|c|c|c|}
\hline \multicolumn{2}{|c|}{ Сомet Brooks* } & \multicolumn{2}{|c|}{ Comet Morehodse } & \multicolumn{2}{|c|}{$\begin{array}{c}\text { CoMET } \\
\text { CUNNINGHAMt }\end{array}$} & \multicolumn{2}{|c|}{$\begin{array}{l}\text { UNIDENTIFIED NU- } \\
\text { CLEAR LINES }\end{array}$} \\
\hline$\lambda$ & Int. & $\lambda$ & Int. & $\lambda$ & Int. & $\lambda$ & Int. \\
\hline 4230 & 0 & & & $423 I . I$ & I $n \ddagger$ & 4230 & I \\
\hline 4238.5 & 0 & $4^{2} 3^{6}$ & $3 \S$ & 4240.4 & $\mathrm{I}-2 n$ & 4238 & I \\
\hline \multirow[t]{2}{*}{$4254 \cdot 5$} & $\circ$ & 4250 & $2 \|$ & $4254 \cdot 3$ & I $n$ & 4255.8 & 2 \\
\hline & $\ldots \ldots$ & 4257 & IO\| & & & & \\
\hline
\end{tabular}

* Long lines, present in head and tail.

$\uparrow$ Especially conspicuous on the spectrograms of December 27, 28, and 3I, I940 (heliocentric distances $0.65,0.63$, and 0.58 ); the lines extend farther from the nucleus than the $\mathrm{CH}$ bands. On the quartz spectrogram of December 28 , I940, showing the tail spectrum, the lines extend far out into the tail.

$\ddagger$ Possibly double at $\lambda_{4229 .}$ (int. $\mathrm{I}$ ) and $\lambda_{423}$ I.8 (int. $\mathrm{I}$ ).

$\S$ Present in head and part of tail.

$1 /$ Present in head and tail; main contributor, $\mathrm{CO}^{+}$.

$\lambda 4050$ and several other unexplained cometary features are due to ionized diatomic molecules. Among these, $\mathrm{NH}^{+}, \mathrm{CN}^{+}$, and $\mathrm{C}_{2}^{+}$would be especially interesting. ${ }^{6}$

We shall see that $\mathrm{CH}^{+}$is present in comets, whereas the case of $\mathrm{OH}^{+}$is doubtful and requires additional observations; $O_{2}^{+}$does not provide any reliable identification.

Identification of $\mathrm{CH}^{+}$in the head and in part of the tail.-Among the unidentified cometary features a group of three lines at $\lambda \lambda{ }_{423} \mathrm{I}, 4238$, and 4254 , appearing in the wavelength range between the $\Delta v=-$ I sequence of $C N$ and the strong $C H$ lines, deserves special attention because it extends, at least partly, into the tail. This group was measured in various comets, especially in Comet Brooks by Wright ${ }^{7}$ and in Comet Morehouse by De la Baume Pluvinel and Baldet. ${ }^{8}$ The measured wave lengths are given in Table I, which contains also my measurements in Comet Cunningham ${ }^{9}$ and the wave lengths of unidentified features listed by Baldet. ${ }^{\text {xo }}$

4 There is a possibility that $\lambda 3934.3$ may be due to $\mathrm{NaH}$ (see McKellar's paper).

5 Additional observational data on the contours of these diffuse lines would be useful.

${ }^{6}$ For the " $\lambda 4050$ group," which consists of a number of discrete, well-separated lines, a molecule with a small moment of inertia is probable. Since the spectrum of $N H^{+}$would be somewhat similar to the isoelectronic radical $\mathrm{CH}$ and since the bands of neutral $\mathrm{NH}$ are fairly strong in comets, there is a very good chance that the $\lambda 4050$ group may be due to $\mathrm{NH}^{+}$. The lines of the $\lambda 4050$ group are not observed as absorption features in late-type stars, and this fact favors also the attribution to an ionized molecule
7 Lick Obs. Bull., 7, 8, r9г2.
9 Unpublished.
${ }^{8}$ Ap. J., 34, 89, I9ri.
ro Ann. obs. astr. phys., Paris, 7, 58, 1926. 
There is little doubt that the lines measured in Comet Morehouse at $\lambda_{42} 5^{\circ}$ and $\lambda_{42} 57$ are mostly due to a band of ${ }^{2} \Pi \rightarrow{ }^{2} \Sigma$ of $\mathrm{CO}^{+}$(comet-tail system), and this will apply to any line measured in the tail near $\lambda_{42}$ 24. But this is not the case in the head, when the other $\mathrm{CO}^{+}$bands of similar intensity are absent. In the head the attribution of $\lambda_{423} 8$ to the $(\mathrm{I}, 2)$ transition of $N_{2}^{+}\left({ }^{2} \Sigma \rightarrow{ }^{2} \Sigma\right)$ is also excluded when the $(0,0)$ band of $N_{2}^{+}$at $\lambda$ 39I4 is not observed. There is a strong transition of $\operatorname{SiN}$ at $\lambda 4^{2} 39$ (system ${ }^{2} \Sigma \rightarrow^{2} \Sigma$, band $[4,4])$; but this cannot contribute appreciably to the observed feature, since the stronger band at $\lambda_{4204}$ is not observed.

The only satisfactory identification that suggests itself is to attribute the three lines to the $(\mathrm{o}, 0)$ band of $\mathrm{CH}^{+}$(system $\left.{ }^{\mathrm{I}} \Pi \rightarrow{ }^{\mathrm{I}} \Sigma\right)$. ${ }^{\mathrm{II}}$ In this case we might also expect a faint $(\mathrm{I}, \mathrm{O})$ band to be present, since in interstellar absorption the $\mathrm{CH}^{+}$molecules reveal their presence by the $R(0)$ lines of the three transitions $(0,0),(I, 0)$, and $(2,0) .{ }^{11}$ The unidentified cometary lines that have been observed in the region between $\lambda 3953$ and $\lambda 3975$ are listed in Table 2 . We majy expect that part of these lines are due to the un-

TABLE 2

GROUP OF UNIDENTIFIED COMETARY LINES NEAR $\lambda 3960$

\begin{tabular}{|c|c|c|c|c|c|}
\hline \multicolumn{2}{|c|}{ Сомeт Brooks* } & \multicolumn{2}{|c|}{ Comet Cunningham $\dagger$} & \multicolumn{2}{|c|}{$\begin{array}{c}\text { UNDENTIFIED NUCLEAR } \\
\text { FEATURES } \ddagger\end{array}$} \\
\hline$\lambda$ & Int. & $\lambda$ & Int. & $\lambda$ & Int. \\
\hline & & $\begin{array}{l}3954.0 \\
3960.2\end{array}$ & $\stackrel{I}{I-2}$ & & \\
\hline 3963 & o & $3963 \cdot 5$ & $\mathrm{I}-2$ & $3962 \cdot 5$ & $\circ$ \\
\hline & $\cdots$ & ......... & $\cdots$ & 3968.4 & 2 \\
\hline 3974 & 0 & $3972 \cdot 7$ & 2 & 3974.8 & $\circ$ \\
\hline
\end{tabular}

* Wright, Lick Obs. Bull., 7, 8, rgr2.

$\dagger$ Swings, Elvey, and Babcock, $A p . J .$, 94, 342, I94I. The lines $\lambda \lambda 3954,3963$, and 3973 were enhanced, together with $\lambda \lambda{ }_{423} \mathrm{I}, 4238$, and 4254 , on the spectrograms of December 27 and $3 \mathrm{I}$, 1940.

$\ddagger$ Baldet, Ann. obs. astr. phys., Paris, 7, 58, 1926.

identified molecules giving rise to the " $\lambda 4050$ group." The $(0,0)$ band of the BaldetJohnson system of $C O^{+}\left(B^{2} \Sigma \rightarrow A^{2} \Pi\right.$, the lower level $A^{2} \Pi$ being the upper state of the comet-tail bands) does not contribute, since the other transitions ( $I, 0)$ and $(0, I)$ of similar intensity are absent. ${ }^{\mathrm{I2}}$

Thus it is suggested that the features near $\lambda 4240$ and $\lambda 3960$ are, at least partly, due to $\mathrm{CH}^{+}$, according to the identifications listed in Table 3 , where the cometary wave lengths given in the first column are average values estimated from Tables I and 2. The $\mathrm{CH}^{+}$molecules are present in the head and in part of the tail.

The observed lines of $\mathrm{CH}^{+}$arise mainly from the rotational levels $J^{\prime}=\mathrm{I}$ and 2. This "low temperature distribution" is similar to that observed for $\mathrm{CH}, \mathrm{NH}, \mathrm{OH}$, and $C N$. Moreover, an examination of the intensity distribution in the exciting solar spectrum ${ }^{13}$ reveals that the absorption transitions to the level $J^{\prime}=3, v^{\prime}=0$, are more reduced by

II I am very grateful to Dr. Herzberg for the privilege of receiving the wave lengths of the $\mathrm{CH}^{+}$lines in advance of publication. For the announcement of the identification of $\mathrm{CH}^{+}$lines in interstellar space see Douglas and Herzberg, $A p . J .$, 94, 381, I94I.

I2 The excitation of $\mathrm{CO}^{+}$molecules to the $B^{2} \Sigma$ level, by solar radiation, would require absorption through the first negative system $B^{2} \Sigma \rightarrow^{2} \Sigma$, around $\lambda$ 2200; we should thus expect the Baldet-Johnson system to be much weaker than the comet-tail spectrum.

I3 Swings, Lick Obs. Bull., r9, I3 I, r94I. 
Fraunhofer lines than those corresponding to $J^{\prime}=2, v^{\prime}=0$. This decreases the intensities of the bright lines $R(2), Q(3)$, and $P(4)$ relative to those of $R(I), Q(2)$, and $P(3)$ in the $(0,0)$ band. The effective slit width for the spectrograms of Comet Cunningham is I.9 A near $\lambda_{4240}$; thus the separation of the $Q(I)$ and $Q(2)$ lines of the $(0,0)$ band is impossible, while $R(0)$ and $R(I)$ may be resolved. The region between $\lambda_{3940}$ and $\lambda 4000$ is complex, whereas that around $\lambda 4240$ contains no lines other than $\lambda \lambda{ }_{42} 3 \mathrm{I}, 4238$, and 4254. The evidence in favor of $\mathrm{CH}^{+}$from the $(\mathrm{O}, \mathrm{O})$ band is, therefore, more convincing than that from the $(\mathrm{I}, \mathrm{O})$ transition. No line was observed in the region of the $(2,0)$ band.

On the whole, the evidence in favor of $\mathrm{CH}^{+}$is satisfactory. Like $\mathrm{CO}^{+}$and $\mathrm{N}_{2}^{+}, \mathrm{CH}^{+}$ molecules are present in the head as well as in the tail, but the extension of $\mathrm{CH}^{+}$into the tail is shorter than that of $\mathrm{CO}^{+}$and $\mathrm{N}_{2}^{+}$.

Discussion of the spectral region $\lambda \lambda 3560-3600$ in comets.-A detäiled investigation of the region around $\lambda 3570$ in cometary spectra is of interest for the following reasons: $(a)$

TABLE 3

$\mathrm{CH}^{+}$LINES IN COMETARY SPECTRA

\begin{tabular}{|c|c|c|c|c|}
\hline \multicolumn{2}{|c|}{ Comets } & \multicolumn{3}{|c|}{ LABORATORY } \\
\hline$\lambda$ & Int. & $\lambda$ & Notation & \\
\hline $423 I^{*}$ & I & $4232 \cdot 54$ & $\mathrm{R}(0)$ & \\
\hline 4238.5 & $I-2$ & $\begin{array}{l}423 \cdot 54 \\
4237 \cdot 56 \\
4239 \cdot 37\end{array}$ & $\begin{array}{l}Q(I) \\
Q(2)\end{array}$ & \\
\hline 4254.4 & I & $4254 \cdot 39$ & $\bar{P}(3)$ & \\
\hline 3954 & I & $\begin{array}{l}3955 \cdot 49 \\
3954 \cdot 37\end{array}$ & $\begin{array}{l}R(1)+R(4) \\
R(2)+R(3)\end{array}$ & \\
\hline 3963 & $I-2$ & $\begin{array}{l}3962.07 \\
3964.26\end{array}$ & $\begin{array}{l}Q(\mathrm{I}) \\
\mathrm{Q}(2)\end{array}$ & $(I, 0)$ \\
\hline 3972 & $I-2$ & 3970.82 & $\mathrm{P}(2)$ & \\
\hline
\end{tabular}

* The two components measured on one spectrogram at $\lambda 4229$.I (int. I) and $\lambda_{423} \mathrm{I} .8$ (int. $\mathrm{I}$ ) would be the $\mathrm{R}(\mathrm{I})$ and $\mathrm{R}(\mathrm{o})$ lines, respectively.

Lines appear which are not due to $\mathrm{CN} ;(b)$ if $\mathrm{OH}^{+}$molecules are present in comets, their $(0,0)$ band should fall in this region (system $3 \Pi \rightarrow 3 \Sigma$ ); and $(c)$ there is an unidentified interstellar line at $\lambda 3579.04,{ }^{14}$ which may be due to a molecule playing a role in comets.

On the basis of the analysis of the $\mathrm{OH}^{+}$spectrum by Loomis and Brandt, ${ }^{\mathrm{I5}}$ it appears that the interstellar line $\lambda 3579.04$ cannot be attributed to $\mathrm{OH}^{+}$. The suspicion that it may be the $\mathrm{R}(0)$ line in the $(3,0)$ band of $\mathrm{CH}^{+}$so far remains unconfirmed, since in the laboratory investigation by Douglas and Herzberg, ${ }^{16}$ in which an attempt was made to observe the emission bands of $\mathrm{CH}^{+}$arising from $v^{\prime}=3$, no band from this level was obtained.

For comparison we shall describe the present situation with respect to cometary spectra in the same region - a situation that is rather confused, owing to the fact that the spectrograms obtained at the McDonald Observatory have a dispersion of only I75 $\mathrm{A} / \mathrm{mm}$ and an effective slit width of $2.5 \mathrm{~A}$ at $\lambda$ 3570. The presence of fairly strong features due to the $\Delta v=+\mathrm{I}$ sequence of $C N$ complicates this spectral region. In Comet

${ }_{4} 4$ This line is not due to the $(\mathrm{I}, \mathrm{O})$ transition of $C N$.

${ }^{15}$ Phys. Rev., 49, 55, I936. $\quad{ }^{6}$ Private communication of Dr. Herzberg. 


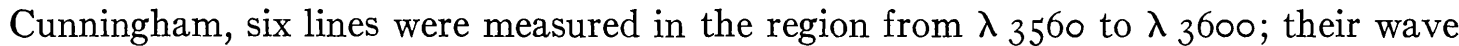
lengths are listed in Table $4 . \quad \lambda 3584$ is undoubtedly the $\mathrm{P}$ branch of the $(\mathrm{I}, 0)$ transition of $C N$, and $\lambda 3577.3$ is essentially a maximum around $\mathrm{R}(5)$ in the $(\mathrm{I}, 0)$ band, with possibly also a contribution of lines around $\mathrm{P}(5)$ in the $(2, \mathrm{I})$ band of $C N . \lambda 3572.2$ seems too strong to be due only to $\mathrm{R}(\mathrm{I} 4)$ of $(\mathrm{I}, \mathrm{O})$ and $\mathrm{R}(5)$ of $(2, \mathrm{I})$, especially since the solar spectrum is appreciably depleted by Fraunhofer lines in the regions of the absorption lines of $C N$ leading to $v^{\prime}=\mathrm{I}, K^{\prime} \sim \mathrm{I}_{5}$. It has been suggested that the abnormal intensity of $\lambda 3572$ might be due to an intensity maximum in the solar spectrum; yet it is also likely that $\mathrm{OH}^{+}$contributes to the intensity of the observed feature. As for $\lambda_{3565}$, it does not seem possible to attribute it to $\mathrm{R}\left(\mathrm{I}_{7}\right)$ of the $(2, \mathrm{I})$ band of $C N$, and it is more probable that it is due to the first lines of the $\mathrm{R}_{3}$ branch of $\mathrm{OH}^{+}$. It should be pointed out that the

TABLE 4

LiNES OBSERVED IN COMET CUNNINGHAM BETWEEN

$\lambda_{3560 \text { AND } \lambda_{3600}}$

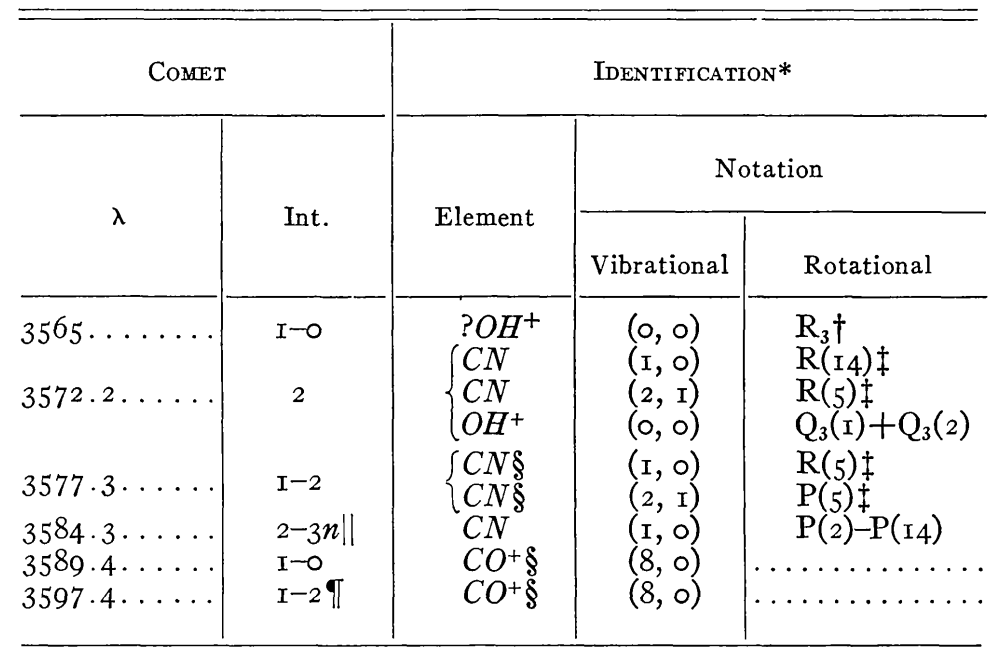

* Contributions by the bands of $N O$ ( $\beta$ system), $N_{2}$ (second positive system) $N^{t}$ (first negative system), and $C_{2}$ (Deslandres-D'Azambuja system) that fall in this region may be disregarded.

$\dagger R_{3}(I), R_{3}(2), R_{3}(3), R_{3}(4)$.

$\ddagger$ Blend of lines around this transition.

$\$$ Minor contribution of $\mathrm{OH}^{+}$not excluded.

|| Extending from $\lambda_{358 \mathrm{r} .4}$ to $\lambda_{3586.3}$.

I Possibly double at $\lambda 3595.7$ and $\lambda$ 3598.6.

solar spectrum contains a number of very strong Fraunhofer lines (especially of $F e$ I and $N i \mathrm{I}$ ) between $\lambda_{3565}$ and $\lambda 3590$, so that the intensity distribution among the rotational lines of the $(0,0)$ band of $O H^{+}$and of the $(\mathrm{I}, 0)$ band of $C N$ must be peculiar as a result of excitation by solar radiation.

The lines $\lambda 3589$ and $\lambda 3597$ were enhanced on the spectrogram of Comet Cunningham, taken on December 28, I94I, on which the tail spectrum was well apparent; this exposure is the only one obtained of Comet Cunningham at the McDonald Observatory, showing all the strong bands of $\mathrm{CO}^{+}$and $\mathrm{N}_{2}^{+}$. It is thus safe to attribute $\lambda_{35} 89$ and $\lambda 3597$ mainly to the $(8,0)$ band of $\mathrm{CO}^{+}$, which has a laboratory intensity of 6 on a scale of $\mathrm{I}-\mathrm{IO}$; but $\mathrm{OH}^{+}$may be a contributor to these features.

Summarizing the present situation, we may say that there is a strong suspicion that the $(\mathrm{O}, \mathrm{O})$ band of $\mathrm{OH}^{+}$is present in cometary spectra. The fainter (I, O), (o, I), and (I, I) bands near $\lambda \lambda 3332,3893$, and 3695, respectively, have not been observed in 
comets. It would be important to observe cometary spectra in the region around $\lambda 3570$ with a higher resolving-power.

Additional data on the $C$ isotopes in comets.-In the case of $C_{2}$, which, unlike $C N, C H$, $\mathrm{NH}, \mathrm{OH}$, and $\mathrm{CH}^{+}$, reveals a rotational intensity distribution corresponding to a fairly high temperature $\left(T \sim 3000^{\circ} \mathrm{C}\right)$, a perturbation of the rotational intensity distribution due to the contour of the exciting solar spectrum might also be expected. Although its detection will require a higher resolving-power than is now available, it is necessary to keep in mind the possible presence of this effect and to exercise great care when searching for the isotopic bands due to $C^{\mathrm{x} 2} C^{\mathrm{x} 3}$ and to $C^{{ }^{13}} C^{{ }^{13}}$, except in the case of the $(\mathrm{I}, \mathrm{O})$ transition.

Bobrovnikoff's conclusion in favor of the presence of the isotopic molecule $C^{\mathrm{r} 2} C^{\mathrm{r} 3}$ in certain comets $^{17}$ was based on two faint bands measured around $\lambda 4744$ and $\lambda{ }_{475} \mathrm{I}$ in comets Brooks and Halley; these bands were attributed to the $(\mathrm{I}, 0)$ transition of $C^{\mathrm{I} 2} C^{\mathrm{I}_{3}}$ and $C^{{ }^{1} 3} C^{{ }^{3}}$. Besides these bands, Brooks's comet revealed also very faint bands measured by Wright ${ }^{18}$ at $\lambda 4723$ and $\lambda 4706$. It is extremely probable that these two features are the $(2, \mathrm{I})$ and $(3,2)$ bands of $C^{\mathrm{I}_{2}} \mathrm{C}^{\mathrm{I}_{3}}$. They cannot be accounted for as peculiar band maxima due to the distortion of the rotational intensity distribution in the $C^{\mathrm{r2}} C^{\mathrm{r2}}$ bands. The evidence for the presence of $C^{x_{3}}$ in comets may thus be strengthened as follows:

$$
\begin{aligned}
& \lambda_{4753} \text { : }(\mathrm{I}, \mathrm{o}) \text { transition of } C^{\mathrm{r}_{3}} C^{13} \\
& \lambda_{4745} \text { : (I, o) transition of } C^{12} C^{13} \\
& \lambda_{4723}:(2, \mathrm{I}) \text { transition of } C^{12} C^{13} \\
& \lambda_{4706:(3,2)} \text { transition of } C^{12} C^{13}
\end{aligned}
$$

\section{GENERAL CONSIDERATIONS ON THE ABUNDANCES OF MOLECULES IN COMETS AND IN INTERSTELLAR SPACE}

Among the molecules which are observed in comets, three diatomic compounds$\mathrm{CH}, \mathrm{CH}^{+}$, and $\mathrm{CN}$-are known to be present in interstellar space. In comets the atmosphere is generated by the liberation of gases occluded in the meteors forming the nucleus; the observed molecules result from the photodissociation of the parent-gases, accompanied or followed by ionization. As for the interstellar molecules, it is much more probable that they result from the association of two atoms. The similarity in constitution between cometary and interstellar gas is a consequence of the cosmic abundances of the elements and of the characteristic physical properties of the molecules.

Certain regions of a cometary head reveal $C H$ molecules, both in the neutral and in the ionized states. We do not know the relative oscillator strengths of $\mathrm{CH}$ and $\mathrm{CH}^{+}$, and no photometric determination has been made of the relative intensities of $\mathrm{CH}$ and $\mathrm{CH}^{+}$ bands. Thus it is impossible to estimate the average ionization of the cometary $\mathrm{CH}$ molecules. But it is certain that the ionization of $C H$ is much lower in comets than in interstellar space. The ionization potentials of $\mathrm{CO}, \mathrm{N}_{2}$, and $\mathrm{CH}$ are, respectively, I4.I \pm 0.2 , I 5.6, and about II volts, thus corresponding, respectively, to the wave lengths $\lambda \lambda 880,800$, and II $30 \mathrm{~A}$. Here, again, we are faced with the absence of data on the relative $f$-values of $\mathrm{CO}^{+}, \mathrm{N}_{2}^{+}$, and $\mathrm{CH}^{+}$. But since the bands due to $\mathrm{CH}^{+}$are weaker than those due to $\mathrm{CO}^{+}$, although the photo-ionization of $\mathrm{CO}$ requires solar radiation of shorter wave length, it may be safely assumed that $C O$ is an abundant molecule in cometary atmospheres and that its abundance must be of the same order as that of $C H$.

At the present time no quantitative theoretical treatment of the formation, dissociation, and ionization of molecules in cometary atmospheres appears possible. The situa-

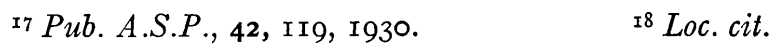


tion is better, however, in the case of the interstellar molecules, although many theoretical questions have not yet been settled. The lines of $\mathrm{CH}, \mathrm{CH}^{+}$, and $\mathrm{CN}$ are rather strong in the spectrum of $\zeta$ Ophiuchi, where $\lambda{ }_{4232.6}$ of $\mathrm{CH}^{+}$has about one-half the intensity of the $\mathrm{H}$ line of $\mathrm{Ca}$ II. $\zeta$ Ophiuchi is far from any O-stars and from the Milky Way, so that most of the $H$ atoms between the star and the sun will be un-ionized, according to B. Strömgren. ${ }^{19}$ For reasons of simplicity we shall use the notations $\left(H^{+}\right)$and $(H)$, respectively, for the interstellar regions where $H$ is mostly ionized and mostly neutral; in Strömgren's theoretical treatment of the ionization of interstellar $H$, the regions $\left(H^{+}\right)$and $(H)$ are rather sharply separated. The fact that the molecular lines are strong in $\zeta$ Ophiuchi, where the atomic lines are not very conspicuous, indicates that the molecular absorption arises mostly in $(H)$ regions. ${ }^{20}$ Most of the stellar radiation beyond $\lambda_{9 \mathrm{I}} 2$ is absorbed in the boundary regions between the $\left(H^{+}\right)$and the $(H)$ regions. But the $C$ atoms will nevertheless be mostly ionized in $(H)$ regions, since the first ionization potential of $C$ is only I I. 20 volts (corresponding to $\lambda$ I IO2) as compared with I 3.53 volts for hydrogen $\left(\lambda_{9}\right.$ I 2$)$. Thus it seems advisable to consider first the formation of $\mathrm{CH}^{+}$ molecules from atoms of $H$ and ionized $C$ in the $(H)$ regions.

Dissociation equilibrium of $\mathrm{CH}^{+}$in interstellar space. - The formation of a $\mathrm{CH}^{+}$molecule requires that the two components $C^{+}$and $H$ approach each other to a distance of the order of I A. If we assume a "kinetic temperature" of $I_{5}, 000^{\circ}$, there will be such an encounter, per cubic centimeter, about once every ro ${ }^{4}$ years. ${ }^{2 I}$ But in order that a molecule may be effectively formed, the excess energy must be removed from the system during the small time $\tau$ of the encounter. In laboratory experiments this removal is generally obtained by means of a third particle. It is easily shown, however, that in interstellar space, where the density of the material is extremely low, such three-body encounters will be too rare to be of any importance (one $C^{+}+H+H$ encounter per cubic centimeter in $\mathrm{IO}^{24}$ years!). For various physical reasons we may exclude also the possibility that the excess energy could be carried away by an electron, leaving a $\mathrm{CH}^{++}$molecule or that the molecule results from the encounter of a normal atom with an excited one. The only process of molecular formation that is sufficiently frequent on the cosmic time scale is the capture of an $H$ atom by a $C^{+}$atom, with simultaneous emission of radiation. The probability of this latter process depends on the energy-curves of the molecular electronic states which the system of two atoms may follow during the encounter. Certain molecules (i.e., $\mathrm{CH}, \mathrm{CH}^{+}$, and $\mathrm{CN}$ ) have excited states dissociating into normal atoms; in such a case, the formation of a molecule may be very effective when the system of the two approaching atoms corresponds to the potential curve of an excited state. ${ }^{22}$ For other molecular formations by capture, the probability may be very small if it requires the emission of a forbidden transition; this is the case of the homonuclear molecule $C_{2}$.

For $\mathrm{CH}, \mathrm{CH}^{+}$, and $C N$, the formation by capture is frequent enough on the cosmic time scale. ${ }^{23}$ Under such conditions we may use the ordinary dissociation formula relative to thermodynamical equilibrium, account being taken of the factor of dilution $\delta$, as was done by Swings and Rosenfeld in I $937 .^{24}$ But the molecular abundance obtained in this way must still be multiplied by a factor $a$, corresponding to the fact that the

${ }^{19} A$ p. J., 89, 526, r939.

${ }^{20}$ This point was stressed by O. Struve, J. Washington Acad. Sci., 31, 2I7, I94I.

${ }^{21}$ The interstellar densities adopted are three $H$ atoms, and $3 \cdot 10^{-3} C^{+}$atoms per cubic centimeter.

${ }^{22}$ According to Dr. G. Herzberg (private communication), "the recombination of $C^{+}+H$ in a twobody collision must occur via the same ${ }^{I} \Pi \rightarrow^{I} \Sigma$ transition that causes the interstellar lines. The upper state ${ }^{I} I I$ dissociates into normal $C^{+}+H$, as does the ground state, and is, in fact, the only state resulting from normal atoms (ions) that can combine with reasonable probability with the ground state."

${ }^{23}$ For comments by $\mathrm{E}$. Teller and $\mathrm{H}$. Beutler regarding this suggestion see the summary of the symposium on interstellar lines at the Yerkes Observatory, on June 30, I94I (Ledoux, loc. cit.).

${ }^{24}$ Ap. J., 86, 483, 1937. 
photodissociation has to start from the lowest rotational, vibrational, and electronic level of the $\mathrm{CH}^{+}$molecule, whereas the formation may lead to any excited state. If we assume with Struve ${ }^{25}$ that $T={ }_{15}, 000^{\circ}$ and $\delta=10^{-16}$, we find, ${ }^{26}$ for the number of $\mathrm{CH}^{+}$ molecules per cubic centimeter, a value comprised between Io $^{-7} \cdot a \cdot N_{H} \cdot N_{C^{+}}$and I0 ${ }^{-8} \cdot a \cdot N_{H} \cdot N_{C^{+}}$. Adopting $N_{H} \cdot N_{C^{+}}=$IO $^{-2}$ (i.e., $N_{H}=3$ and $N_{C^{+}}=3$. IO $^{-3}$ ), we find for $N_{\mathrm{CH}^{+}}$a value between IO $^{-9} \cdot a$ and ${ }^{-10}{ }^{-10} \cdot a$ per cubic centimeter. There is some uncertainty in the values of $T$ and $\delta$, but the order of magnitude of $N_{C^{+}}$must be correct.

As was previously stated, the correction factor $a$ is due to the fact that practically all the $\mathrm{CH}^{+}$molecules are on their lowest rotational level, which slows down the photodissociation, as compared with the capture process. Dr. R. Oppenheimer has obtained an approximate value for the correction factor $a .{ }^{27}$ When two atoms collide, they will in general have a relative angular momentum that can be estimated from the mass, relative velocity, and distance of collision. They will essentially keep this angular momentum if, during the collision, capture takes place. For this reason capture takes place into a large number of rotational states. The number of such states is the correction factor $a$, and its value is

$$
a=\frac{E \times I}{\hbar^{2}}
$$

where

$$
E=\text { energy of collision, } I=\text { moment of inertia, } \hbar=h / 2 \pi(h=\text { Planck's constant }) \text {. }
$$

For a kinetic temperature of about $15,000^{\circ} \mathrm{C}$, the factor $a$ is very nearly $\mathrm{IO}^{3}$ for $\mathrm{CH}^{+}$. The final computed value for the abundance of $\mathrm{CH}^{+}$is thus somewhere between Io $^{-6}$ and I0 ${ }^{-7}$ per cubic centimeter.

In view of the various uncertainties involved in the calculation, the result is gratifyingly close to the value expected from the observations. The interstellar abundances of $C H$ and $C N$ have been estimated by Dunham, who gives the following values:

$$
N(C H) \sim 2.10^{-6} / \mathrm{cm}^{3}, \quad N(C N) \sim{ }_{10^{-6}} / \mathrm{cm}^{3} .
$$

Although $\lambda 4_{4232.6}$ of $\mathrm{CH}^{+}$is of about the same intensity as $\lambda 4300.3$ of $\mathrm{CH}$, we have no idea of the relative oscillator strengths of $\mathrm{CH}^{+}$and $\mathrm{CH}$, so that the abundance of $\mathrm{CH}^{+}$ cannot readily be estimated. Dunham's values of $N(\mathrm{CH})$ and $N(\mathrm{CN})$ are also uncertain, and its seems reasonable to suggest simply an abundance of the same order for $\mathrm{CH}^{+}$. The theoretical treatment outlined here provides also a calculated value of the same order.

Ionization equilibrium of $\mathrm{CH}^{+}$and $\mathrm{CH}$ in interstellar space.- The next step consists in the comparison of the abundance of the $C H$ molecule in its normal and in its ionized states. In the $\left(H^{+}\right)$regions, most of the $C H$ molecules will be in the doubly ionized state; ${ }^{28} C H^{+}$will be more abundant than $C H$, but it will be less abundant than in $(H)$ regions, where $\mathrm{CH}^{++}$and the higher stages of ionization are absent. We are thus justified in considering the ionization equilibrium of $C H$ in the $(H)$ regions only.

${ }_{25}$ Proc. Nat. Acad. Sci., 25, 36, 1939.

${ }^{26}$ The computation was made with an energy of dissociation of 3.5 volts for $\mathrm{CH}^{+}$; a value of this order seems plausible by comparison with the isoelectronic molecule $B H$ and with $C H$. The accurate value $D\left(\mathrm{CH}^{+}\right)=3.84 \mathrm{v}$. obtained by Herzberg and Douglas became available to the author only after completion of the present paper.

${ }^{27}$ Private communication. I want to thank Dr. Oppenheimer for his help in connection with the determination of the interstellar abundances of molecules.

${ }^{28}$ No spectroscopic data are available on the doubly ionized diatomic molecules; laboratory work is desirable, especially on the doubly ionized molecules consisting of two light, cosmically abundant atoms. 
The ionization potentials of $C$ (II.20 volts) and $C H^{29}$ ( $\sim$ I I volts) are not very different and are in any case smaller than the ionization potential of hydrogen. Most of the stellar radiation of wave length shorter than $\lambda$ 9I 2 is absent, so that the ionization of $C$ and $\mathrm{CH}$ can arise only from the absorption of stellar radiation in the wave-length range $\lambda \lambda$ I I00-9 2 A. Except for the ionization corrections similar to the dissociation factor $a$ and for the differences in statistical weights, we should expect the abundance ratio of $\mathrm{CH}^{+}$and $\mathrm{CH}$ to be very similar to that of $\mathrm{C}^{+}$and $C$.

If we assume that no $H$ atom is ionized in the $(H)$ regions, the same must be true for $H e, N, O$, and $N e$, which have higher ionization potentials than hydrogen. Hence the electron density must come primarily from $C$, and secondarily from $S, C l$, and the abundant metals. Under such conditions, the electron density $N_{e}$ must be of the order of $10^{-2}$ per cubic centimeter, or smaller. Adopting the values of $T$ and $\delta$ suggested by Struve $^{30}$ or by Greenstein ${ }^{3 \mathrm{I}}$ for the spectral region around $\lambda$ Iroo A, we obtain for the ionization ratio $N\left(\mathrm{CH}^{+}\right) / N(\mathrm{CH})$ a value between $2 \times \mathrm{Io}^{3}$ and $2 \times 10^{5}$. By what factor the ionization is reduced, because of the fact that no radiation beyond $\lambda$ 9 2 A is available, cannot readily be determined. On the other hand, the factor of ionization reduction for electron captures into excited electronic states is rather complicated. According to Dr. Oppenheimer, ${ }^{32}$ the ionization correction does not reach such large values as the dissociation correction. It is known that in the atomic cases heretofore considered, the correction factor for ionization is of the order of 3 -IO. In any case it seems difficult to reconcile the very high ionization ratio $N\left(\mathrm{CH}^{+}\right) / N(\mathrm{CH})$, obtained from the ionization equation on the assumption that $N_{e}=10^{-2} / \mathrm{cm}^{3}$, with the fact that the $C H$ lines are not much weaker than the $\mathrm{CH}^{+}$lines. Even if an extreme total-correction factor of roo be assumed, $N\left(\mathrm{CH}^{+}\right)$would still be at least twenty times larger than $N(\mathrm{CH})$. This would require that the $\mathrm{CH}^{+}$molecule have a very low $f$-value and consequently an abundance of at least $4 \times 10^{-5} / \mathrm{cm}^{3}$. Such a high molecular abundance seems theoretically impossible.

From the general evidence collected, it appears that the erroneous assumption concerns the electron density $N_{e}$. If this were multiplied by a factor of about ro or somewhat larger, the computed results would be in agreement with the observations. The ionization of $\mathrm{CH}$ would then be decreased by approximately the right amount, and most of the $C$ atoms would still be ionized. An increase of electron density to about $N_{e} \sim \mathrm{IO}^{-\mathrm{I}}$ $\mathrm{cm}^{3}$ would mean that about Io per cent of the $H$ atoms are still ionized in the regions which would be pure $(H)$ regions in Strömgren's simple picture.

Dunham has compared the intensities of $\lambda 4300.3$ of $C H$ and $\lambda{ }_{4232.6}$ of $\mathrm{CH}^{+}$in various stars. ${ }^{33}$ In $\zeta$ Ophiuchi the strength of $\lambda 4_{42} 32.6$ is high, being almost half as strong as the $\mathrm{H}$ line of $C a$ II and stronger than $\lambda$ 4300.3. In other stars, i.e., $\chi^{2}$ Orionis, the intensity ratio of $\lambda_{4233}$ and $\lambda_{4300}$ is reversed. This indicates that the ionization conditions are not constant in regions where the molecular absorption originates. Dunham stated also that the intensities of $\lambda 4232.6$ of $\mathrm{CH}^{+}$and of $\lambda 4300.3$ of $\mathrm{CH}$ do not appear to be correlated with the intensities of $N a$ I or $C a$ I. This is not surprising, since the absorption lines of $\mathrm{Na}$ I and $\mathrm{Ca}$ I originate mostly in $\left.\mathrm{H}^{+}\right)$regions, whereas the molecular lines originate mostly in $(H)$ regions. The intensities of the interstellar molecular lines are probably better correlated with space reddening than the atomic lines of $C a \mathrm{I}, C a \mathrm{II}, N a \mathrm{I}, K \mathrm{I}$, and $F e \mathrm{I}$; but the $T i$ II lines, originating mostly in $(H)$ regions, should behave like the molecules.

${ }^{29} \mathrm{G}$. Herzberg and A. E. Douglas have obtained $D\left(\mathrm{CH}^{+}\right)=3.84$ e.v.; from this, $D(C H)$ and $I(C)$, they found $I(\mathrm{CH})=$ I0.9 e.v., with an error that is very probably less than 0.2 e.v. (private communication of Dr. Herzberg).

${ }^{\circ 0}$ Proc. Nat. Acad. Sci., 25, 36, r939; Gerasimovič and Struve, Ap. J., 69, I9, I929.

${ }^{31}$ Unpublished. $\quad{ }^{2}$ Private communication.

33 Proc. Amer. Phil.Soc., 81, 28 I, г939; Pub. A.S.P., 49, 26, г937. 
The ionization problem is quite different in a cometary atmosphere. Photo-ionization by solar radiation is probably the mechanism for the formation of $\mathrm{CO}^{+}, \mathrm{N}_{2}^{+}$, and $\mathrm{CH}^{+}$ from $C O, N_{2}$, and $C H$. But because of the low density and the small extension of the cometary regions common to $\mathrm{CH}$ and $\mathrm{CH}^{+}$, there is practically no capture of electrons by $\mathrm{CH}^{+}$ions. In other words, once a $\mathrm{CH}$ molecule has been able to absorb photoelectrically a solar photon of wave length shorter than $\lambda$ I roo A, there is practically no chance for the resulting $\mathrm{CH}^{+}$ion to return to the neutral state by electron capture.

In stellar atmospheres of late type that are rich in $\mathrm{CH}$, the presence of $\mathrm{CH}^{+}$would hardly be expected, since in these cool atmospheres where the condition of thermodynamic equilibrium more or less obtains, few if any $\mathrm{CH}$ molecules would be ionized.

Dissociation equilibrium of $C N$ and $C_{2}$ in interstellar space.-The $C N$ molecules are mostly formed in the ionized state $C N^{+}$in $(H)$ regions, since most of the $C$ atoms are ionized and most of the $N$ atoms are neutral. The physical data that would be required for a discussion of the ionization and dissociation equilibria of $C N$ and $C N^{+}$are the energies of dissociation $D_{0}(C N)$ and $D_{0}\left(C N^{+}\right)$of $C N$ and $C N^{+}$in their ground electronic level, and the ionization potential $I(C N)$. These three data are related by the cycle

$$
I(C N)=D_{0}(C N)+I(C)-D_{0}\left(C N^{+}\right)
$$

There is still some uncertainty with respect to $D_{0}(C N)$; Herzberg ${ }^{34}$ suggests $D_{0}(C N)$ $=5.96$ volts, but larger and smaller values have also been considered. Nothing is known of $D_{0}\left(C N^{+}\right)$; the isoelectronic molecule $C_{2}$ has an energy of dissociation $D_{0}\left(C_{2}\right)=3.6$ volts, and a value of this order should be expected for $D_{0}\left(C N^{+}\right)$. No accurate direct measure of $I(C N)$ is available. Only an approximate value $\mathrm{I}_{4} \pm 2$ volts was obtained by Kallmann and Rosen by the method of collisions of the second kind. ${ }^{35}$ But the uncertainty of this value is too large even for a qualitative discussion of the ionization problem, since the essential point is to know whether $I(C N)$ is larger or appreciably smaller ${ }^{36}$ than the ionization potential of hydrogen, I3.53 volts. If we adopt $D_{0}(C N)=5.96$ volts, equation (I) shows that we have $I(C N)<I(H)$, if $D_{0}\left(C N^{+}\right)<3.63$ volts. It is clear that until additional laboratory investigations on $D_{0}(C N)^{+}$or $I(C N)$ are carried out, we shall not be able to discuss the case of interstellar cyanogen in detail. Not all the interstellar $C N$ molecules are on the lowest rotational level $K^{\prime \prime}=0$; but the population on the second level $K^{\prime \prime}=\mathrm{I}$ is already small compared with $K^{\prime \prime}=0$. Hence the dissociation correction will be large, especially since the moment of inertia of $C N$ is about eight times that of $\mathrm{CH}$.

Although the Swan bands constitute the main source of visual light in cometary heads, no interstellar line of $C_{2}$ has ever been found. ${ }^{37}$ That interstellar absorption features due to $\mathrm{C}_{2}$ should be very much weaker than the $C H$ lines results from the following considerations.

I. The formation of $C_{2}$ molecules resulting from the association of two neutral $C$ atoms will be rare, owing to the low abundance of carbon compared to hydrogen and to the further fact that most of the interstellar $C_{2}$ atoms are ionized. Also the probability of formation of the homonuclear $C_{2}$ molecule during a collision of two neutral $C$ atoms is much smaller than for $C H$ or $C N$.

2. If homonuclear molecules like $C_{2}$ exist in interstellar space, their distribution in the rotational levels will probably differ from that of heteronuclear molecules of similar moment of inertia like $C N$. This point was recently mentioned by the author ${ }^{38}$ and stressed

34 Molecular Spectra and Molecular Structure, I, 485, r939.

35 Zs. f. Phys., 6r, 332, I930.

${ }^{36}$ If $I(C N)$ is only slightly smaller than $\mathrm{I} 3.53$ volts, only a narrow spectral range of stellar radiation will be available to ionize $C N$.

37 Dunham, private communication.

${ }^{38}$ J.R.A.S. Canada, 35, 77, 1941. 
by Teller. ${ }^{39}$ The average lifetime of a molecule like $C H$, in a rotational level $K^{\prime \prime}$, is about I09 times longer than on an excited electronic level (approximate proportionality of the lifetimes to $\nu^{-3}$ ); it is thus about Io-roo seconds. For $C N$ it would be still longer, around IO ${ }^{3-10^{4}}$ seconds. For a homonuclear molecule like $C_{2}$ the average lifetime would be Io 4 seconds, multiplied by the ratio of the probability of dipolar emission to that of quadrupolar emission of a rotational line. This may lead to an average lifetime of the same order as the cosmic time scale. Consequently, for homonuclear molecules, a larger number of rotational levels will be populated than for a heteronuclear molecule of similar moment of inertia. Each $\left(v^{\prime}, 0\right)$ band of $C_{2}$ would therefore be expected to show a large number of rotational lines $4^{\circ}$ instead of three or less as in the cases of $\mathrm{CH}^{+}, \mathrm{CH}$, and $C N$.

The fact that the $C_{2}$ molecules would be distributed among a number of rotational levels reduces also the probability of discovering their interstellar lines. Finally, the dissociation correction factor would be smaller in such a case.

On the basis of the above theoretical considerations we are justified in concluding that interstellar lines of $C_{2}$, if they are ever observed, must be much weaker than those of $\mathrm{CH}$, $\mathrm{CH}^{+}$, and $C N$. With a low dispersion, the blends of $C_{2}$ lines should appear like diffuse features.

I wish to thank most sincerely the director and the members of the staff of the Lick Observatory for their cordial hospitality and for numerous helpful discussions.

Lick ObSERVATORY

December I, I94I

${ }^{39}$ See n. 22.

${ }^{40}$ If these homonuclear molecules could have a fairly high abundance, they would be able to explain diffuse lines of the type observed in the visual region. 